\title{
Two experts, three opinions: volatile organic compounds' testing methods and regulative systems
}

\author{
Daniel-Johann Huber ${ }^{1} \cdot$ Julia Huber $^{1} \cdot$ Franziska Hesser $^{1} \cdot$ Eva Höllbacher $^{2}$ \\ Tobias Stern ${ }^{3}$
}

Received: 20 February 2017 / Published online: 24 October 2017

(C) The Author(s) 2017 2017. This article is an open access publication

\begin{abstract}
As a result of the implementation of the CEmarking in the European Union, the testing method as defined in prEN 16516 is about to become the new volatile organic compounds (VOC) testing standard and replace a multitude of testing methods and regulative systems in Europe that assess the existence of VOC in building materials, including wood-based materials. The aim of this study is, first, to compile expert positions regarding testing methods and regulative systems for VOC in wood-based materials, and second, to examine the suitability of using an Argument-Delphi approach to point out consent or dissent regarding testing methods for VOC. A systematic content analysis of initial interviews with 13 experts produced 130 arguments which were subscribed to 13 categories, resulting in 6 potential positions of which 5 were actively taken by experts. While the second step was undertaken as a cross verification of the analysis, the experts were asked to choose a final position as a last step. It was observed that several experts changed their initial position to testing methods and regulative systems for VOC, but still without a consensus arising within the expert panel. Hence, the results of the study reveal that no verifiable consent exists within the group of experts-neither concerning testing methods, nor
\end{abstract}

Tobias Stern

tobias.stern@uni-graz.at

1 Market Analysis and Innovation Research Team, Kompetenzzentrum Holz (Wood K plus), Feistmantelstrasse 4, Vienna, Austria

2 Indoor Quality and Emission Control Team, Kompetenzzentrum Holz (Wood K plus), Institute of Chemical Engineering, Vienna University of Technology, Getreidemarkt 9, 1060 Vienna, Austria

3 Institute of Systems Sciences, Innovation and Sustainability Research, University of Graz, Merangasse 18, Graz, Austria regulative systems. The Argument-Delphi approach applied constitutes a useful method in pointing out dissent between the experts towards a legal requirement like a European standard.

\section{Introduction}

The European population is estimated to spend approximately $90 \%$ of their time indoors (Schweizer et al. 2006). Indoor air quality has become an important issue with regard to health concerns (Jones 1999). Improvements in building insulation for the sake of saving energy led to tighter building envelopes, which aggravate the indoor air exchange and the accumulation of pollutants in indoor air (Zhang and Smith 2003). Indoor air is influenced by indoor climate conditions, such as temperature, humidity, light conditions or noise level (AgBB 2015) and by various indoor and outdoor pollutants, such as $\mathrm{CO}, \mathrm{CO}_{2}$, particulate matter and volatile organic compounds (VOC) (Salthammer and Bahadir 2009). These pollutants are released by a high number of possible natural and anthropogenic sources including construction materials which are an important indoor source of VOC (Wolkoff 2013; Buchter et al. 2002; Lin et al. 2009). Wood and wood-based products which are widely used as construction materials also emit specific VOC (Zhu and Shen 2016; $\mathrm{Yu}$ and Kim 2012) and thus affect indoor air quality (He et al. 2012; Kim et al. 2010).

Some emissions of construction materials, including certain VOC are related to illnesses (Jones 1999) like the sick building syndrome or even cancerous disease (Buchter et al. 2002; Guo et al. 2004), and therefore pose a risk to human health (Derbez et al. 2014). No significant differences in VOC air concentrations based on the use of different materials (wood vs non-wood) was found in Nyrud 
et al. (2012). Further studies showed that some wood-based building materials act as diffusion barriers or as emission sinks (Niedermayer et al. 2013; Höllbacher et al. 2016).

For this reason, tests are conducted to measure VOC emissions from construction products (Eichholzer et al. 2010; Ojala et al. 2006). These tests are mostly conducted according to the specifications defined in the standards of the ISO $16000 \mathrm{ff}$ series. The main test set-ups are inert test chambers as defined in ISO 16000-9 (DIN 2006). The construction products regulation (CPR) of the European Union (EU), which is effective since July 2013, is the starting point for international VOC regulative systems. The CPR demands that "construction works be designed and executed so as not to endanger the safety of persons, domestic animals or property nor damage the environment" (The European Parliament and the Council of the European Union 2011). In particular, this may not occur by "emissions of dangerous substances, volatile organic compounds (VOC), greenhouse gases or dangerous particles into indoor or outdoor air" (The European Parliament and the Council of the European Union 2011). However, the specification of the regulative systems is still reserved to individual member states (The European Parliament and the Council of the European Union 2011). Compulsory VOC regulations can be found nowadays in Germany, France and Belgium (Eurofins 2016).

In the foreseeable future, VOC emission tests on construction products will be required to obtain the $\mathrm{CE}$ label (Oppl 2014)—which requires a harmonized testing method at EU level (Oppl 2014). A proposed new horizontal testing method is based on the ISO 16000 series and was first defined in the technical specification CEN/TS 16516 which is now published as draft standard prEN 16516 "Construction products - assessment of release of dangerous substances-determination of emissions into indoor air" (Oppl 2014).

The decision on a horizontal testing method for Europe is one of the requirements for including VOC emissions in the CE label of a product or product group. Furthermore, the individual harmonized product standards have to be adapted to include VOC measurements. The subsequent assessment of VOC emissions requires either a European regulation on VOC or, if such a regulation is nonexistent, national assessment criteria. Once these requirements are fulfilled the testing method as defined in the prEN will become obligatory also in the wood-processing industry (Oppl 2014). When testing for VOC emissions of wood and wood-based materials problems arise. VOC emissions from wood products vary depending on the processed wood species and the production process (Jensen et al. 2001). Further obstacles for testing result from the inhomogeneous character of the natural material, which make it difficult to establish reproducible test results (Eichholzer et al. 2010; Yrieix et al. 2010). Due to these difficulties, the use and viability of the standardised testing methods as basis for possible regulative systems for wood products is heavily discussed. Therefore, the objective of this study is gathering assessments to identify consent and dissent within the expert panel regarding testing methods and regulative systems of VOC emissions from wood-based materials. A consensus could demonstrate which testing method and which regulative system in the EU is actually preferred by experts. Therefore, research questions investigated are:

- Which testing methods and regulative systems for the determination of VOC of wood-based materials are considered appropriate by experts?

- To what extent is Argument Delphi suitable to point out consent or dissent within the expert panel?

\section{Materials and methods}

To investigate the research questions, a Delphi approach (Häder 2009; Linstone and Turoff 1975; Stern et al. 2012; Sutterlüty et al. 2017) was chosen to conduct a survey among experts from relevant industries and research organisations. The Delphi method is an explorative and multistep survey technique, based on a panel of experts, which are iteratively questioned and confronted with results of former steps. The anonymity of the participants and the feedback are the fundamental elements of the Delphi method (Gordon 2009).

Argument Delphi is a variant of the Delphi method to aggregate ideas (Ammon 2005). The Argument Delphi according to Kuusi (1999) builds on a multistage classification system and presents different lines of argumentation. For the expert panel, it is not the sample size but the coverage of a broad range of expertise which is essential in Argument Delphi (Rikkonen and Tapio 2009). Argument Delphi is not only capable of depicting consent but also dissent. This is relevant because the divergence in an expert panel can also point out relevant issues (Rikkonen and Tapio 2009). In this study, the Argument Delphi is conducted in three consecutive rounds, beginning with expert interviews, followed by data verification by the experts and finalizing with the expert's positioning.

State of the art literature regarding VOC emission measurement and testing methods (e.g. AgBB 2015; Derbez et al. 2014; DIN 2006; Eichholzer et al. 2010; Eurofins 2016; Guo et al. 2004; He et al. 2012; Höllbacher et al. 2017; Jensen et al. 2001; Jones 1999; Kim et al. 2010; Lin et al. 2009; Niedermayer et al. 2013; Nyrud et al. 2012; Ojala et al. 2006, Oppl 2014; The European Parliament and the Council of the European Union 2011; Wolkoff 2013; Yu and Kim 2012; Zhu and Shen 2016) was reviewed prior to the development of the interview guideline. A pre-test with researches active 
in the field of VOC research helped to verify the interview guideline.

Thereafter, potential experts for the panel were identified based on knowledge and experience. To create a broad range of expertise, it was the aim to integrate various relevant sectors into the survey: wood-processing industry, private research institutions, state research institutions, university research institutions and measuring institutes. The selection of experts was based on the subsequent literature research as well as on recommendations of experts who confirmed their participation (snowballing). Thus, a list of 60 experts was compiled, from which 13 experts were willing to participate. The participating experts came from France (4), Austria (3), Germany (2), Belgium (1), Finland (1), Poland (1) and Sweden (1) representing wood-processing industry (2), private research institutions (2), state research institutions (4), university research institutions (3) and measuring institutes (2).

After completing the preparatory actions, the Argument Delphi exploration was conducted in the period from July until October 2014 either face-to-face, by telephone or via Skype call. The study proceeded in three rounds according to Kuusi (1999):

\section{- Delphi round 1: expert interview}

In the first round, assessments were conducted in form of qualitative expert interviews with each of the participating experts, using a semi-narrative semi-structured interview to enable queries, to control the flow of conversation and to give the interviews structure and comparability (Häder 2009; Kruse 2014; Meuser and Nagel 2009). The interview guideline was sent to the experts in advance. Thereafter, the interviews of the first round were transcribed, their arguments were categorised, reduced content wise and summarised using the content analysis software MAXQDA.

\section{- Delphi round 2: data verification by the experts}

The intermediate results (summary of round 1) were sent out to all experts to inform them about all arguments stated and to verify the arguments. The experts would give feedback, if some arguments were misinterpreted. The arguments were densified and thereof, six different positions were derived (see Table 2). Every expert was assigned to one position due to the statements given during the expert interview (first round).

\section{- Delphi round 3: experts' positioning}

The six positions were presented in a decision tree (see Fig. 1). This figure was sent to all participating experts, who were asked to decide on their positions. They also had the possibility to revise or change their former assessment.

\section{Results}

In the first Delphi round, 13 experts were interviewed. In total 130 arguments were derived by their interviews, which were subdivided into 13 thematic categories like "harmonized testing method", "limit values" or "excluding woodbased materials from VOC regulations" (see Table 1). On average, ten arguments were given by each interviewed expert.

After conducting the first and the second Delphi round, the arguments were condensed and summarised. From 13 argument categories, five different expert positions were developed (see Table 2). Position 6 (harmonized testing method with a harmonized classification system) was not explicitly expressed by the experts arguments, but can be derived as a logical combination of possible arguments and is therefore mentioned to complete the range of positions.

With the arguments and statements of the experts, each expert was assigned to a position. Due to the fact that the positions described in Table 2 follow a logic hierarchy of typical yes-no-decisions, a four stage decision tree was developed (see Fig. 1). In the third Delphi round, the experts could decide which position they prefer, using the developed decision tree.

Figure 2 shows the experts' positions during the first and the third Delphi round. Four experts stuck to their position, six experts changed their position (indicated by the bold arrow) and three experts opted out of the survey (indicated by the lined arrow). The changes can be analysed considering the expert origin as well as their sector. Overall, the high rate of turnover on country as well as on sector level is surprising. A partial explanation for the rather high turnover rate might be the arrangement of the survey: in round 1 , the experts are caught in their position and do not think of another position. In round 3, the decision tree gives an overview of all possible positions and enables the opportunity to choose the most preferred position. An according hypothesis can be supported by the fact that, for example, all French experts initially agreed on one position in round 1 , and spread in round 3. Furthermore, the experts of the industrial sector disagreed in round 1 , but concurred in round 3 .

The results show further, that it was not possible to reach consent with the participating experts regarding a specific testing method or a regulative system for measuring VOCs in wood-based materials.

Regarding testing methods, the following expert assessments were conducted: several experts appreciate a harmonized testing method. They see cost benefits for the wood-processing industry due to the fact that multiple tests disappear. However, not all of the experts judge the prEN 16516 to be appropriate for testing VOCs in wood-based materials, and recommend therefore a standardised test chamber size, which is large enough (at least $1 \mathrm{~m}^{3}$ ) to get 


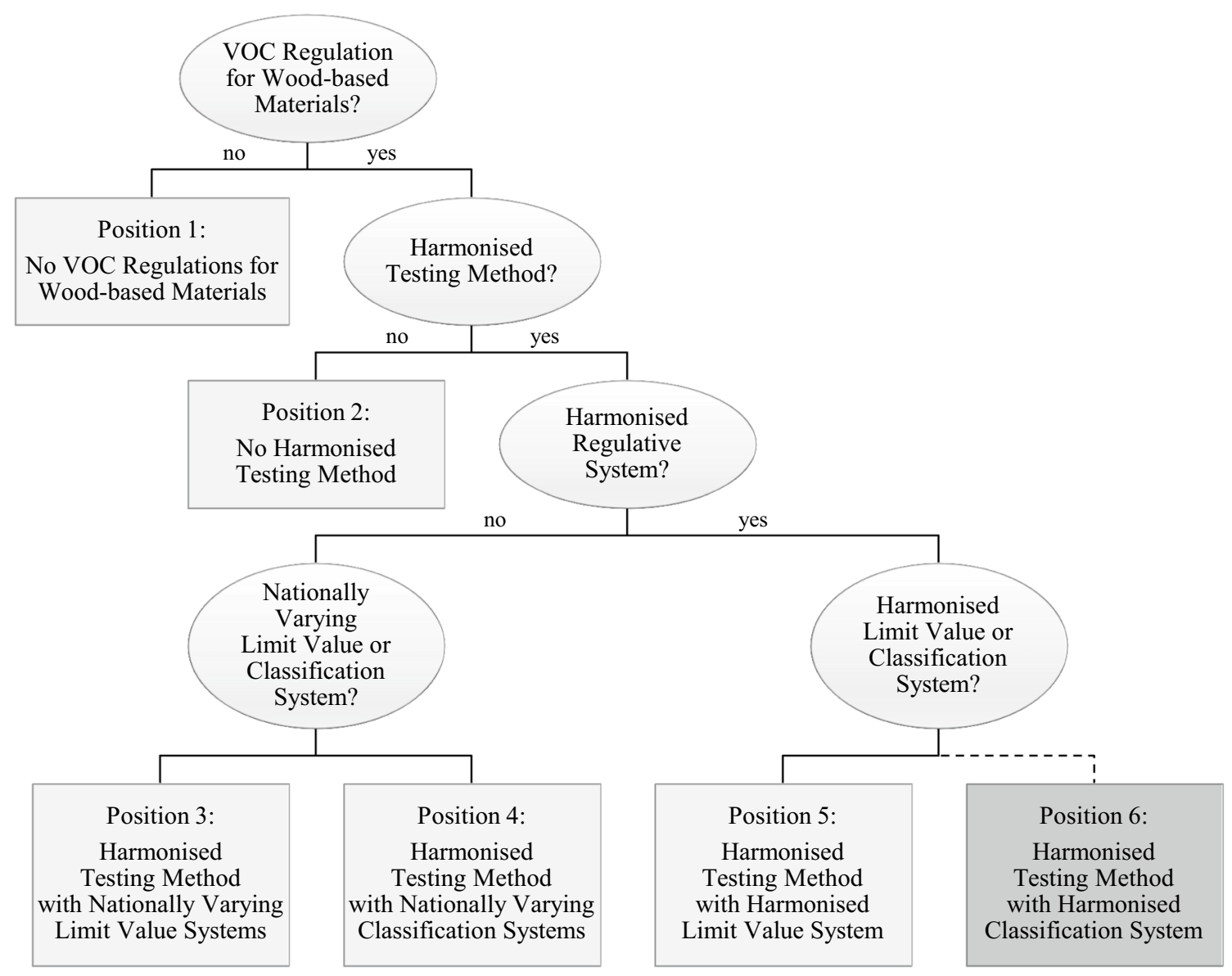

Fig. 1 Decision tree derived from expert positions described in Table 2

Table 1 Categorization of 130 arguments provided by 13 experts during the first Delphi round

\begin{tabular}{lc}
\hline Argument category & $\begin{array}{c}\text { Number of arguments } \\
\text { and statements }(\Sigma=130)\end{array}$ \\
\hline Excluding wood-based materials from VOC regulations & 18 \\
Difficulty in implementation of VOC regulations in general & 4 \\
Criticism regarding customers & 1 \\
Regulative systems in the EU & 14 \\
Criticism regarding regulative systems in the EU & 12 \\
Harmonised testing method & 36 \\
Criticism regarding chamber testing & 5 \\
Criticism regarding chamber size & 1 \\
Toluene equivalent vs original response & 5 \\
Lowest concentration of interest (LCI) and toxicology & 6 \\
Analytics & 3 \\
Limit values & 16 \\
Global testing methods & 3 \\
\hline
\end{tabular}

reproducible test results of inhomogeneous wood-based materials in different laboratories. For the same reason, they propose a broader range for the parameters humidity and temperature. Some experts were concerned that the harmonised testing method is not perfectly oriented on the market needs, as it is an entirely political issue. They also criticised that the mandatory completion of the testing 
Table 2 Expert positions (1-6) derived from the categorization of arguments provided during the first Delphi round

Pos. Description Selected arguments of the position

1 No VOC regulations for wood-based materials

2

No harmonised testing method

3 Harmonised testing method with nationally varying limit value systems

4

Harmonised testing method with nationally varying classification systems

Harmonised testing method with harmonised limit value system
Emissions from wood-based materials are natural. There is no toxicological verification for TVOC values in wood-based materials

Wood is a natural emitter and this fact cannot simply be changed by product development. There are people, who react sensible to individual emissions, but only a few. Simply banning these products, just because they emit more than stone, would be unwise

The harmonised testing method has numerous flaws. Inter laboratory tests have shown, that the results of different laboratories are quite different. This is not only because of the chamber sizes, but also the fault of analytics. The problem is not an inaccurate work of the employees, but a fundamental weakness of the testing method

The problem with chamber testing is that it is not possible to use emission measurement to indicate real concentrations within woodbased materials. Considering this, emission testing of wood-based materials can only be seen as indication

I prefer a harmonised testing standard, which has to base on a technically justified basis and must not be solely a political issue

I don't think there is a need to unify limit values. Different countries have their individual regulations based on their climate, conditions and culture

A harmonised testing method in the EU is preferable and beneficial for the market. Regarding regulations, I think that the individual member states of the EU should set their own limit values, depending on each one's climate and culture

The French system is appealing, because it lacks the "German stubbornness". In Germany a product is approved or rejected. Within the French system, the market and therefore consumers have the ability to decide for themselves whether or not to acquire a specific product

I am not fond of limit values because they restrict the consumer's ability to decide freely

We do need a European "overall system". A harmonised testing system is the first step towards this goal. Now we need an evaluation system and following a limit value system. The European commission already went towards this by proposing LCI values for several substances

The Joint Research Centre (JRC) of the European Commission has already harmonised a number of limit values of different member states. These are implemented in the new Belgium regulation. The JRCs list of harmonised limit values is shorter than the mandatory list of Germany. Therefore, the Belgium regulation uses the German values complementary

No arguments were named for this position method for prEN 16516 leaves no more time for important adjustments.

Regarding regulative systems, the French system "Émissions dans l'air intérieur" received several positive arguments like the ability of the costumer to decide about a product purchase and not a categorical prohibition of a product when exceeding the limit values. In addition, the better comparability of the emission values within laboratories appeals to the experts. The German AgBB system is being perceived as comprehensive and precise due to its requirements to testing and limit value method, but, compared to the French regulative system, the comparability of inter laboratory tests is missed.

However, it is important to note that these perceptions are based on the individual statements of the experts interviewed in 2014. The comparability of inter laboratory tests for example may have been subject to experiments lately and due to such scientific results expert's perceptions may change. 


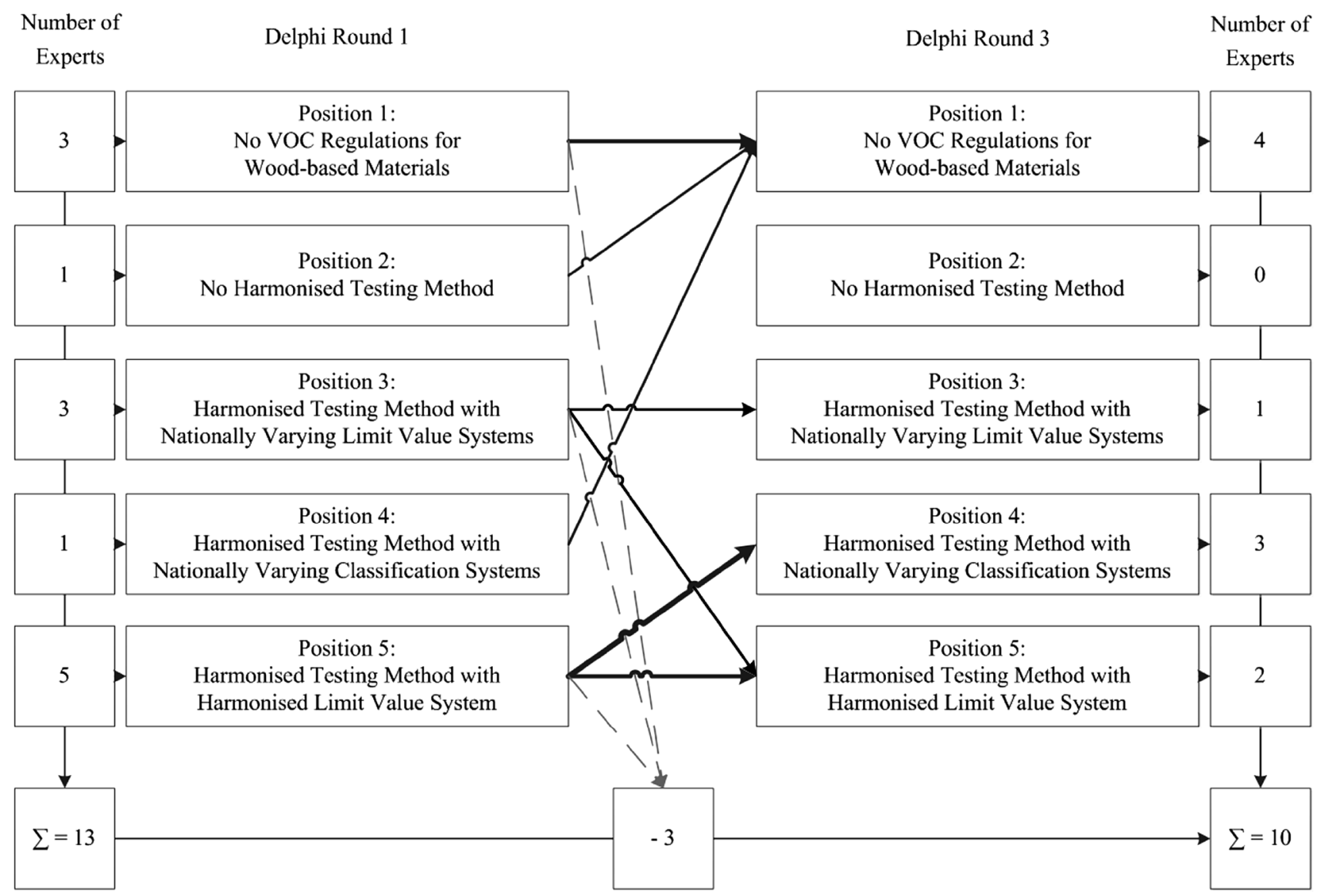

Fig. 2 Expert positions of Delphi rounds 1 and 3

\section{Discussion and conclusion}

The three-step Argument-Delphi approach applied in this study produced two main results. The development of the decision tree on expert positions regarding VOC regulation illustrates the first result. Given the controversial discussions on this topic, its value is based on the systematic structure referring to underlying arguments. The second notable result is the observation that several experts decided to change their predominant positions after being confronted with the decision tree (a broader range of possible positions). Based on the 13 participating experts no systematic pattern behind expert positions was observed considering nationality or professional (sector) background. Hence, the results of the study reveal, that no verifiable consent exists between the experts-neither concerning testing methods, nor regulative systems. A participation in a survey like the one presented in this study might be a source of information and facilitate learning about different positions. Clear positioning requires a clear opinion on something, hence opinions may not yet have been clear in this learning stage. While other studies applying argument Delphi have already reported frequent changes in expert opinions (Makkonen et al. 2016), the present study could not observe the frequently reported tendency to adjust opinion to majorities, maybe because of a lack of clear majorities in the first round.

As with every expert-based research design, limitations regarding sample selection must be taken into account. A different or larger set of experts may have led to other results in particular regarding frequencies in particular positions. Therefore, the absolute and relative frequencies observed in particular positions in the first or third round shall not be overrated. However, the general structure of the decision tree as well as the pattern of experts changing positions is unlikely affected by other or larger panels as long as expert diversity is considered. As a matter of theoretical saturation the number of additional experts to be considered in order to add further branches to the decision tree can be expected as disproportional. However, in this study no arguments were found to derive the complementary position number 6 (harmonized testing method with harmonized classification system) in the first round. Even in the second round no claim was made to include such a position in the survey. Hence, this position was not covered in the third round but it was included in the decision tree as a matter of completeness. Nevertheless the decision tree can be taken as a perfect 
starting point for further research in technical, social and policy related research disciplines.

The Argument-Delphi approach, although seldom applied to such a technical context (Rodgers et al. 2016), has been found a useful method in pointing out dissent between the experts with regard to a legal requirement like a European standard. The research design used in this study could be replicated some years after the implementation of prEN 16516 to evaluate how such an implementation may affect expert opinions in the medium term.

Acknowledgements Open access funding provided by University of Graz. This work was supported by the Austrian Research Promotion Agency (FFG) under the COMET program Grant Number 844608.

Open Access This article is distributed under the terms of the Creative Commons Attribution 4.0 International License (http://creativecommons.org/licenses/by/4.0/), which permits unrestricted use, distribution, and reproduction in any medium, provided you give appropriate credit to the original author(s) and the source, provide a link to the Creative Commons license, and indicate if changes were made.

\section{References}

AgBB (Ausschuss zur gesundheitlichen Bewertung von Bauprodukten) (2015) Vorgehensweise bei der gesundheitlichen Bewertung der Emissionen von flüchtigen organischen Verbindungen (VOC und SVOC) aus Bauprodukten (Procedure for the sanitary valuation of volatile organic compounds (VOC and SVOC) of construction products). https://www.umweltbundesamt.de/sites/default/files/ medien/355/dokumente/agbb-bewertungsschema_2015_2.pdf. Accessed 10 Jan 2017

Ammon U (2005) Delphi-Befragung (Delphi survey). In: Kühl S (ed) Quantitative Methoden der Organisationsforschung: Ein Handbuch (Quantitative methods of organisational research: a manual). VS Verlag für Sozialwissenschaften, Wiesbaden, pp 115-138

Buchter A, Ecker C, Jablonsky M, Staab J (2002) Innenraumbelastung und Sick Building Syndrom (Indoor air pollution and sick building syndrome). Institut und Poliklinik für Arbeitsmedizin der Universität des Saarlandes, Homburg/Saar

Derbez M, Berthineau B, Cochet V, Lethrosne M, Pignon C, Riberon J, Kirchner S (2014) Indoor air quality and comfort in seven newly built, energy-efficient houses in France. Build Environ $72: 173-187$

DIN (2006) Indoor air - part 9: determination of the emission of volatile organic compounds from building products and furnishingemission test chamber method. International Standard Organization, Geneva

Eichholzer A, Niedermayer S, Aschacher G (2010) VOC_Flüchtige organische Stoffe (VOC-volatile organic compounds). Zuschnitt Zeitschrift über Holz als Werkstoff und Werke in Holz 39:22

Eurofins (2016) Belgian regulations on VOC emissions from construction products. http://www.eurofins.com/consumer-product-testing/ information/compliance-with-law/european-national-legislation/ belgian-regulation-on-voc-emissions/. Accessed 10 Jan 2017

Gordon T (2009) The Delphi method. Futures research methodology: the Millennium Project: version 3.0

Guo H, Lee SC, Chan LY, Li WM (2004) Risk assessment of exposure to volatile organic compounds in different indoor environments. Environ Res 94(1):57-66
Häder M (2009) Delphi-Befragungen: Ein Arbeitsbuch (Delphi surveys: a workbook), 2nd edn. VS Verlag für Sozialwissenschaften, Wiesbaden

He Z, Zhang Y, Wei W (2012) Formaldehyde and VOC emissions at different manufacturing stages. Build Environ 47:197-204

Höllbacher E, Ters T, Rieder-Gradinger C, Srebotnik E (2016) Influences of interior fitting processes on the indoor air quality of two model rooms. Eur J Wood Prod 74(5):693-701

Höllbacher E, Ters T, Rieder-Gradinger C, Srebotnik E (2017) Emissions of indoor air pollutants from six user scenarios in a model room. Atmos Environ 150:389-394

Jensen LK, Larsen A, Mølhave L, Hansen MK, Knudsen B (2001) Health evaluation of volatile organic compounds (VOC) emissions from wood and wood-based materials. Arch Environ Health 56(5):419-432

Jones AP (1999) Indoor air quality and health. Atmos Environ 33(28):4535-4564

Kim S, Choi YK, Park KW, Kim JT (2010) Test methods and reduction of organic pollutant compound emissions from woodbased building and furniture materials. Bioresour Technol 101(16):6562-6568

Kruse J (2014) Qualitative Interviewforschung-Ein integrativer Ansatz (Research with qualitative interviews - an integrative approach). Beltz Juventa, Weinheim

Kuusi O (1999) Expertise in the future use of generic technologies: epistemic and methodological considerations concerning Delphi studies. HeSe Print, Helsinki

Lin C-C, Kuo-Pin Y, Zhao P, Lee G, W-M (2009) Evaluation of impact factors on VOC emissions and concentrations from wooden flooring based on chamber tests. Build Environ 44(3):525-533

Linstone HA, Turoff M (1975) The Delphi method. Techniques and applications. Addison-Wesley, Reading

Makkonen M, Hujala T, Uusivuori J (2016) Policy experts' propensity to change their opinion along Delphi rounds. Technol Forecast Soc Change 109:61-68

Meuser M, Nagel U (2009) Das Experteninterview—konzeptionelle Grundlagen und methodische Anlage (The expert interview-conceptual foundations and methodical approach). In: Pickel S, Pickel G, Lauth H-J, Jahn D (eds) Methoden der vergleichenden Politikund Sozialwissenschaft (Methods of the comparative political and social sciences]. VS Verlag für Sozialwissenschaften, Wiesbaden, pp 465-479

Niedermayer S, Fürhapper C, Nagl S, Polleres S, Schober KP (2013) VOC sorption and diffusion behavior of building materials. Eur J Wood Prod 71(5):563-571

Nyrud A, Bringslimark T, Englund F (2012) Wood use in a hospital environment: VOC emissions and air quality. Eur J Wood Prod 70(4):541-543

Ojala S, Lassi U, Keiski R (2006) Testing VOC emission measurement techniques in wood-coating industrial processes and developing a cost-effective measurement methodology. Chemosphere 62(1):113-120

Oppl R (2014) New European VOC emissions testing method CEN/TS 16516 and CE marking of construction products. GefahrstoffeReinhaltung der Luft 74(3):62-68

Rikkonen P, Tapio P (2009) Future prospects of alternative agrobased bioenergy use in Finland-constructing scenarios with quantitative and qualitative Delphi data. Technol Forecast Soc Change 76:978-990

Rodgers M, Booth A, Norman G, Sowden A (2016) Research priorities relating to the debate on assisted dying: what do we still need to know? Results of a modified Delphi technique. BMJ Open. https:// doi.org/10.1136/bmjopen-2016-012213

Salthammer T, Bahadir M (2009) Occurrence, dynamics and reactions of organic pollutants in the indoor environment. Clean Soil Air Water 37(6):417-435 
Schweizer C, Edwards RD, Bayer-Oglesby L, Gauderman WJ, Ilacqua V, Juhani Jatunen M, Lai HK, Nieuwenhuijsen M, Kunzli N (2006) Indoor time-microenvironment-activity patterns in seven regions of Europe. J Expo Sci Environ Epidemiol 17(2):170-181

Stern T, Heil G, Ledl C, Schwarzbauer P (2012) Identifying innovation barriers using a Delphi method approach: the case of technical lignin in the wood-based panel industry. Int Wood Prod J 3(2):116-123

Sutterlüty A, Hesser F, Schwarzbauer P, Schuster KC, Windsperger A, Stern T (2017) A Delphi approach to understanding varying expert viewpoints in sustainability communication: the case of water footprints of bio-based fiber resources. J Ind Ecol 21:412-422

The European Parliament and the Council of the European Union (2011) Regulation (EU) No 305/2011. lying down harmonised conditions for the marketing of construction products and repealing. Off J Eur Union 88(5):1-10
Wolkoff P (2013) Indoor air pollutants in office environments: assessment of comfort, health, and performance. Int J Hyg Environ Health 216(4):371-394

Yrieix C, Dulaurent A, Laffargue C, Maupetit F, Pacary T, Uhde E (2010) Characterization of VOC and formaldehyde emissions from a wood based panel: results from an inter-laboratory comparison. Chemosphere 79(4):414-419

Yu CWF, Kim JT (2012) Long-term impact of formaldehyde and VOC emissions from wood-based products on indoor environments; and issues with recycled products. Indoor Built Environ 21(1):137-149

Zhang JF, Smith KR (2003) Indoor air pollution: a global health concern. Br Med Bull 68:209-225

Zhu XD, Shen YLJS (2016) Volatile organic compounds (VOCs) emissions of wood-based panels coated with nanoparticles modified water based varnish. Eur J Wood Prod 74:601-607 